Textures and Microstructures, 1987, Vol. 7, pp. 187--205

Photocopying permitted by license only

(C) 1987 Gordon and Breach Science Publishers Inc.

Printed in the United Kingdom

\title{
On the Representation of Texture Functions of Cubic Metals in Euler Space
}

\author{
P. VAN HOUTTE
}

Department of Metallurgy and Materials Engineering, Katholieke Universiteit Leuven, Belgium

(Received 22 January 1987; in final form 5 February 1987)

Cubic-orthorhombic texture functions (e.g., of rolling textures) are conventionally represented by Euler diagrams with a range of $0^{\circ}$ to $90^{\circ}$ for $\phi_{1}, \Phi$ and $\phi_{2}$. Some drawbacks of this method are discussed. By means of inverse pole figure representations, it is explained how this range can be subdivided into three completely equivalent "unit zones". In order to overcome the drawbacks mentioned above, without adding yet a new type of O.D.F. representation to the list of those that already exist, it is proposed to continue using the same type of representation but to consider only unit zone 3 , which is much less distorted than the two other unit zones. It is recommended to show the limits between the unit zones in the Euler diagrams.

KEY WORDS: Orientation distribution function, metric of the Euler space, equivalent zones, cubic symmetry, inverse pole figures.

\section{INTRODUCTION}

Euler angles have been widely used for the description of crystal orientations since Bunge and Roe introduced O.D.F. analysis (Bunge, 1982). There are however some disadvantages to the representation of texture functions in Euler space. Equal distances in Euler space do not correspond to equal angular distances between crystal orientations. It is not easy to see the relationship between pole figures and inverse pole figures and an O.D.F. represented in Euler space. The part of Euler space that is 
customarily used for the representation of rolling textures of cubic metals contains three times the same information.

The purpose of the present paper is to give a short discussion of these problems, and to show how they can be dealt with without having to introduce yet another method for the graphical representation of crystal orientations.

\section{THE CONVENTIONAL EULER DIAGRAMS}

Figure 1 shows the Euler angles as defined by Bunge (1982). Roe's angles will not be considered here. The figure shows how the reference system of the sample can be rotated in order to obtain the reference system of the crystal. The angles $\phi_{1}, \Phi$ and $\phi_{2}$ describe this rotation. A right-hand orthogonal coordinate system is used for both reference systems.

In the most general case (no crystal symmetry and no sample symmetry), the following ranges should be considered for these

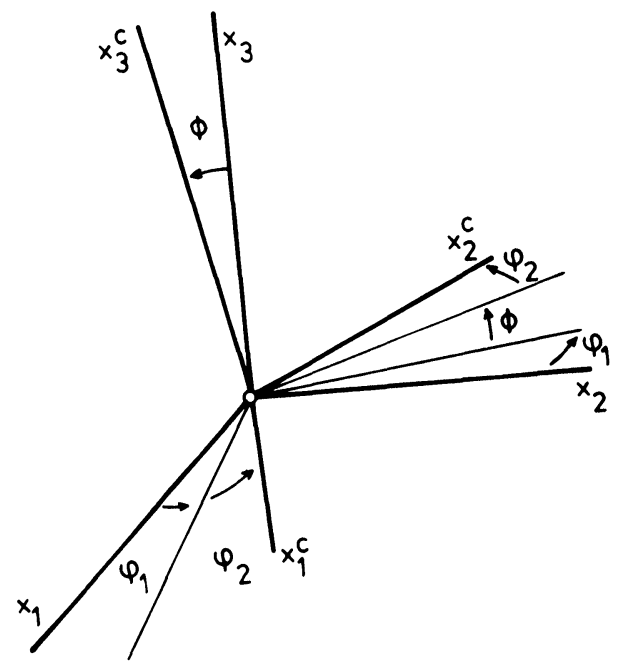

Figure 1 Euler angles used for the description of a crystal orientation. $\left\langle x_{i}\right\rangle$ are the sample axes; for example: $x_{1}=$ rolling direction, $x_{3}=$ normal direction. $\left\langle x_{i}^{c}\right\rangle$ are the coordinate axes of the crystal system, e.g. the $\left\langle\begin{array}{lll}1 & 0 & 0\end{array}\right)$ axes in case of cubic crystal symmetry. 
Euler angles:

$$
\begin{aligned}
& 0 \leq \phi_{1}<360^{\circ} \\
& 0 \leq \Phi<180^{\circ} \\
& 0 \leq \phi_{2}<360^{\circ}
\end{aligned}
$$

Crystal and sample symmetry both considerably reduce the minimum range that must be considered in order to describe a texture. Consider for example the case of cubic crystal symmetry. There are 24 equivalent ways to put a right-handed orthogonal coordinate system on the $\left\langle\begin{array}{lll}1 & 0 & 0\end{array}\right\rangle$ axes of a crystal. This leads to 24 different sets of Euler angles all describing the same crystal orientation, hence strongly reducing the minimum range in Euler space. In addition to that, rolled samples are conventionally thought to possess 3 twofold symmetry axes, namely the rolling direction $\left(\mathrm{RD}=\mathrm{x}_{1}\right)$, the transverse direction $\left(\mathrm{TD}=\mathrm{x}_{2}\right)$ and the normal direction $\left(\mathrm{ND}=\mathrm{x}_{3}\right)$. Mark that these axes are fixed with respect to the sample reference system, not with respect to the crystal reference system. They reduce the minimum range of the Euler angles by a factor 4 .

For the representation of rolling or annealing textures of cubic metals, most authors use the following range for the Euler angles:

$$
\begin{aligned}
& 0 \leq \phi_{1} \leq 90^{\circ} \\
& 0 \leq \Phi \leq 90^{\circ} \\
& 0 \leq \phi_{2} \leq 90^{\circ}
\end{aligned}
$$

This "conventional" range still contains three times the same information, as will be explained below.

Figure 2 shows an example of a graphical representation of an O.D.F. in this range. 19 sections $\phi_{2}=$ constant are shown, as is customary for rolling or annealing textures of f.c.c. metals. (For b.c.c. metals, sections $\phi_{1}=$ constant are used.) In each of these sections, a $\phi_{1}-\Phi$ subspace is shown in which contour lines indicate the value of the texture function (or O.D.F.) $f(g)$. Each section has a Cartesian coordinate system. Its origin is at the upper left corner. From there on, the $\phi_{1}$-axis points to the right and the $\Phi$-axis downwards. Thus Euler space is graphically represented as if it were a Euclidian space. The advantage obviously is that the measurement 


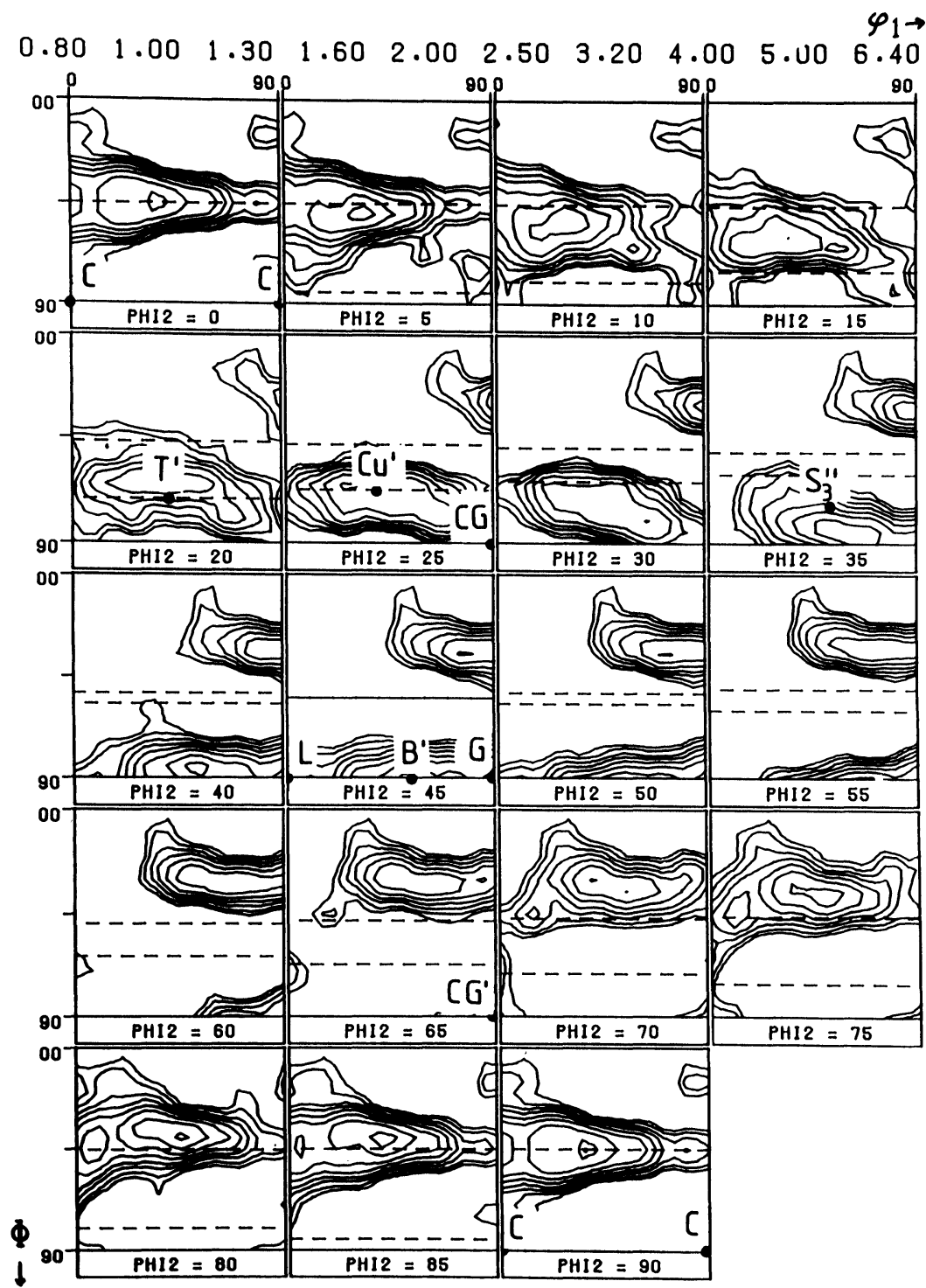

Figure 2 Texture function (or orientation distribution function) of a moderately rolled brass sample. 19 sections of the type $\phi_{2}=$ constant are shown. 
of the Euler angles of a particular texture component is very easy in such a diagram. However, the Euler space is not Euclidian. This means that equal distances seen in Euler space do not necessarily correspond to equal "differences" between crystal orientations in physical space. Consider for example the singularity at $\Phi=0$. It is seen in Figure 1 that the crystal orientation that is obtained by rotating the reference system of the sample by an angle $\Omega$ around $\mathrm{x}_{3}$ is as $v$ ell described by the Euler angles $\phi_{1}=\Omega, \Phi=0$ and $\phi_{2}=0$ as by the Euler angles $\phi_{1}=0, \Phi=0$ and $\phi_{2}=\Omega$. Obviously only the sum $\phi_{1}+\phi_{2}$ matters in this case. As a result, each crystal orientation for which $\Phi=0$ has an infinite number of representations in Euler space. Another way to express the "curved" character of Euler space is to look at its "volume" element, which is

$$
\mathrm{d} g=\frac{1}{8 \pi^{2}} \sin \Phi \mathrm{d} \phi_{1} \mathrm{~d} \Phi \mathrm{d} \phi_{2}
$$

instead of merely being $d \phi_{1} d \Phi d \phi_{2}$ (Bunge, 1982). Sin $\Phi$ can be considered as a measure of the distortion of Euler space: the closer it is to zero, the worse the distortion.

\section{EULER SPACE AND INVERSE POLE FIGURE REPRESENTATION}

Van Houtte and Aernoudt (1976) have discussed the sample symmetry of deformation textures of cubic metals in terms of inverse pole figures. Their inverse pole figures differ from conventional ones in this sense that they are primarily used to represent individual crystal orientations instead of axial densities. The orientation of the axes of the sample with respect to the crystal axes are represented in these inverse pole figures, with emphasis on the axis $x_{3}$. The position of the representation of the axis $x_{3}$ only depends on the Euler angles $\phi_{2}$ and $\Phi$, not on $\phi_{1}$ (Figure 3), as has also been pointed out by Pospiech (1972). The latter represents an additional rotation around $x_{3}$, in order to bring the sample axes $x_{1}$ and $x_{2}$ in their positions with respect to the crystal axes.

Rolling textures have an orthorombic sample symmetry. This has been called class $B$ by the above authors. According to their results, the minimum range required for the description of such texture can 

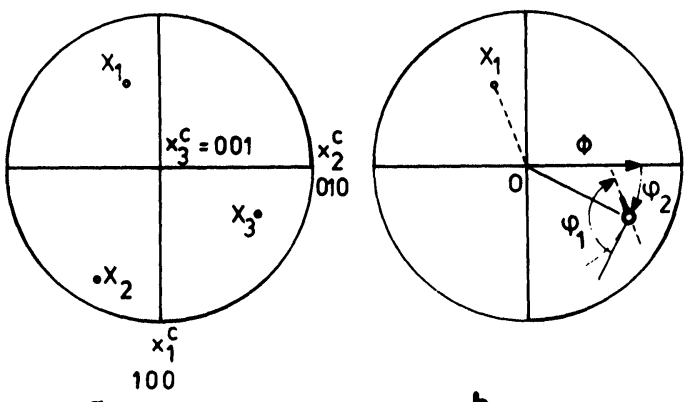

a

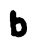

Figure 3 Inverse pole figure representation of a crystal orientation (example for cubic metals). (a) The three specimen axes are plotted in a (001) standard projection. (b) The representation of the axis $x_{3}$ depends only on the Euler angles $\Phi$ and $\phi_{2}$, used as polar coordinates. This is also true for non-cubic lattice symmetries.

be described in an inverse pole figure; in the case of cubic lattice symmetry it would be (Figure 4):

- for $\mathrm{x}_{3}$ : one unit triangle such as $001-011-111$ or $100-110-111$. This sets a range for $\phi_{2}$ and $\Phi$;

- for $\phi_{1}: 0-180^{\circ}$.

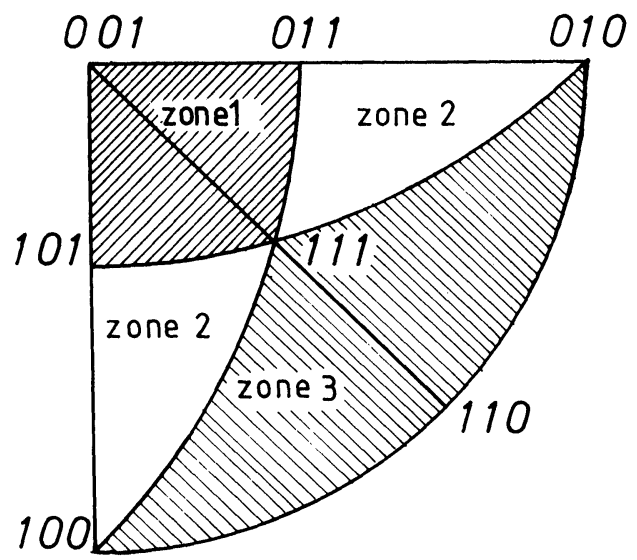

Figure 4 Part of the inverse pole figure for cubic crystal symmetry. The three unit zones to which Tables 1 and 2 refer are shown. 


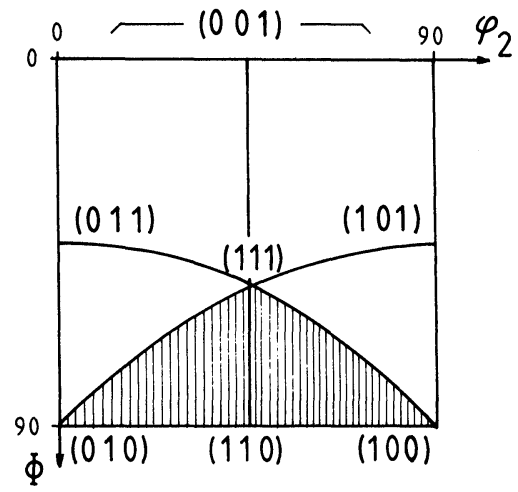

Figure 5 A $\phi_{1}=$ constant section in Euler space. Each point of this figure represents a combination $\left(\Phi, \phi_{2}\right)$ and so corresponds to a point in an inverse pole figure such as Figures 3-4. The shaded area represents unit zone 3.

The ranges for other sample symmetries can also be found in this paper.

In a subsequent paper (Gil Sevillano, Van Houtte and Aernoudt, 1980) the above authors described the correspondence between inverse pole figure representations and Euler space more explicitly. Figure 5 is taken from this paper. It shows a $\phi_{1}=$ constant section of Euler space. In such plot, $\phi_{2}$ and $\Phi$ merely are Cartesian coordinates instead of being polar coordinates as in the inverse pole figure (Figures 3-4). Hence, by making abstraction of $\phi_{1}$, such figure can very well be used to represent the orientation of the sample axis $\mathrm{x}_{3}$ with respect to the crystal. Figures 4 and 5 clearly show the point-by-point correspondence between the two types of representations. There is one singularity: the point 001 in the inverse pole figure becomes a whole line segment in Figure 5.

In this second paper, the authors also derived an alternative minimum range for textures with cubic crystal symmetry and orthorombic sample symmetry (class B). First they showed that the following symmetry operation is allowed in that case:

-invert the $\mathrm{x}_{2}$-axis; note that the $\mathrm{x}_{1} \mathrm{x}_{2} \mathrm{x}_{3}$ system is transformed from a right handed system into a left handed system by doing so;

- the entire $x_{1} x_{2} x_{3}$-system is now reflected into the mirror plane (1 $\overline{1} 0$ ), which transforms it again into a right handed (and thus allowed) system. 
The entire procedure can also be described by using rotational symmetry elements only (both of the crystal and of the sample).

Let $\phi_{1}, \Phi$ and $\phi_{2}$ be the Euler angles before application of this symmetry operation. They are transformed into

$$
\begin{aligned}
& \phi_{1}^{*}=180^{\circ}-\phi_{1} \\
& \Phi^{*}=\Phi \\
& \phi_{2}^{*}=90^{\circ}-\phi_{2}
\end{aligned}
$$

by it. Application of this symmetry operation allows the transformation of the minimum range given above into the following range:

- for $\mathrm{x}_{3}$ : two unit triangles occupying symmetrical positions with respect to the mirror plane ( $1 \overline{1} 0)$, for example 001-011-111 and 001-101-111;

- for $\phi_{1}: 0-90^{\circ}$.

Let us consider three possible choices for the range for $\mathrm{x}_{3}$ (Figure 4):

(1) the triangles 001-011-111 and 001-101-111;

(2) the triangles 010-011-111 and 100-101-111;

(3) the triangles 010-110-111 and 100-110-111.

Consider now all three of those ranges together. It is seen in Figures 4 and 5 that this combined range completely fills up the triangle 001-010-100 and that it corresponds to the range for the Euler angles given by Eqs. $(2 \mathrm{a}-\mathrm{c})$, i.e. the conventional range. It is now quite clear that the latter contains three mutually equivalent minimum ranges. Table $1 \mathrm{a}-\mathrm{c}$ lists the Euler angles of some important crystal orientations for each of them.

It is clear that the presence of three minimum ranges in the conventional range for rolling textures of cubic metals may lead to confusion when reading an Euler diagram. It is strongly recommended to consider only one of the three minimum ranges when for example counting the number of different texture components.

The unit ranges mentioned above have also been described by Pospiech (1972) and by Hansen, Pospiech and Lücke (1978). They can very easily be adapted to other sample symmetries. Assume for example cubic crystal symmetry and monoclinic sample symmetry 
Table 1 Euler angles of the most common texture components of the rolling textures of f.c.c. and b.c.c. metals. (a) representations for which $x_{3}=N D$ lies in unit zone 1 , consisting of the triangles (001)-(011)-(111) and (001)-(101)-(111). (b) representations for which $x_{3}=$ ND lies in unit zone 2, consisting of the triangles (010)-(011)-(111) and (100)-(101)-(111). (c) representations for which $\mathrm{x}_{3}=$ ND lies in unit zone 3 , consisting of the triangles (010)-(110)-(111) and (100)-(110)-(111).

Column (A) lists crystal orientations that are all physically different from each other. In some groups, they can however be derived from each other by $180^{\circ}$ rotations around a twofold rotation symmetry axes of the SAMPLE (i.e., ND, RD or TD). Such orientations are separated by a dashed line. In other cases, the orientations are separated by a full line.

The indicial notations of column (B) represent the same crystal orientations as those of column (A). Among the 24 possible descriptions of a given orientation, those for which the $x_{3}$-axis falls within the considered unit zone are given. Two indicial notations of column (B) that are not separated by a dashed or a full line represent the same crystal orientation. When separated by a dashed line, they indeed represent different crystal orientations, which however can be derived from each other by a $180^{\circ}$ rotation around one of the twofold symmetry axes of the sample (a)

\begin{tabular}{|c|c|c|c|c|c|}
\hline \multirow[t]{2}{*}{ Code } & \multirow[t]{2}{*}{ (A) } & \multirow[t]{2}{*}{ (B) } & \multicolumn{3}{|c|}{ Euler angles } \\
\hline & & & $\phi_{1}$ & $\Phi$ & $\phi_{2}$ \\
\hline $\mathrm{C}$ & $\left(\begin{array}{lll}0 & 0 & 1\end{array}\right)\left[\begin{array}{lll}1 & 0 & 0\end{array}\right]$ & 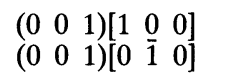 & $\begin{array}{r}0 \\
90\end{array}$ & $\begin{array}{l}0 \\
0\end{array}$ & $\begin{array}{l}0 \\
0\end{array}$ \\
\hline CG & $\left(\begin{array}{lll}0 & 2 & 1\end{array}\right)\left[\begin{array}{lll}1 & 0 & 0\end{array}\right]$ & 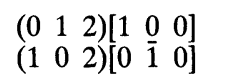 & $\begin{array}{l}0 \\
0\end{array}$ & $\begin{array}{l}26.56 \\
26.56\end{array}$ & $\begin{array}{r}0 \\
90\end{array}$ \\
\hline G & $\left(\begin{array}{lll}0 & 1 & 1\end{array}\right)\left[\begin{array}{lll}1 & 0 & 0\end{array}\right]$ & $\begin{array}{l}\left(\begin{array}{lll}0 & 1 & 1\end{array}\right)\left[\begin{array}{lll}1 & 0 & 0\end{array}\right] \\
\left(\begin{array}{lll}1 & 0 & 1\end{array}\right)\left[\begin{array}{lll}0 & 1 & 0\end{array}\right]\end{array}$ & $\begin{array}{l}0 \\
0\end{array}$ & $\begin{array}{l}45 \\
45\end{array}$ & $\begin{array}{r}0 \\
90\end{array}$ \\
\hline B & $\left(\begin{array}{lll}0 & 1 & 1\end{array}\right)\left[\begin{array}{lll}2 & \overline{1} & 1\end{array}\right]$ & 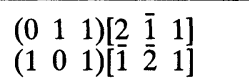 & $\begin{array}{l}35.26 \\
35.26\end{array}$ & $\begin{array}{l}45 \\
45\end{array}$ & $\begin{array}{r}0 \\
90\end{array}$ \\
\hline $\mathrm{S}_{3}$ & $\left(\begin{array}{lll}\overline{1} & \overline{2} & \overline{3}\end{array}\right)\left[\begin{array}{lll}6 & 3 & \overline{4}\end{array}\right]$ & $\left(\begin{array}{lll}2 & 1 & 3\end{array}\right)\left[\begin{array}{lll}\overline{3} & \overline{6} & 4\end{array}\right]$ & 58.98 & 36.7 & 63.43 \\
\hline $\mathrm{T}$ & $\left(\begin{array}{lll}4 & 4 & 11\end{array}\right)\left[\begin{array}{lll}11 & \overline{11} & 8\end{array}\right]$ & $\left(\begin{array}{lll}4 & 4 & 11\end{array}\right)\left[\begin{array}{lll}\overline{11} & \overline{11} & 8\end{array}\right]$ & 90 & 27.21 & 45 \\
\hline $\mathrm{Cu}$ & $\left(\begin{array}{lll}1 & 1 & 2\end{array}\right)\left[\begin{array}{lll}\overline{1} & \overline{1} & 1\end{array}\right]$ & $\left(\begin{array}{lll}1 & 1 & 2\end{array}\right)\left[\begin{array}{lll}\overline{1} & \overline{1} & 1\end{array}\right]$ & 90 & 35.26 & 45 \\
\hline $\mathrm{H}$ & $\left(\begin{array}{lll}0 & 0 & 1\end{array}\right)\left[\begin{array}{lll}1 & 1 & 0\end{array}\right]$ & $\left(\begin{array}{lll}0 & 0 & 1\end{array}\right)\left[\begin{array}{lll}1 & \overline{1} & 0\end{array}\right]$ & 45 & 0 & 0 \\
\hline $\mathrm{K}^{\prime}$ & $\left(\begin{array}{lll}0 & 0 & 1\end{array}\right)\left[\begin{array}{lll}3 & 2 & 0\end{array}\right]$ & $\left(\begin{array}{lll}0 & 0 & 1\end{array}\right)\left[\begin{array}{lll}2 & \overline{3} & 0\end{array}\right]$ & 56.31 & 0 & 0 \\
\hline $\mathrm{K}$ & $\left(\begin{array}{lll}0 & 0 & 1\end{array}\right)\left[\begin{array}{lll}3 & 2 & 0\end{array}\right]$ & $\left(\begin{array}{lll}0 & 0 & 1\end{array}\right)\left[\begin{array}{lll}3 & 2 & 0\end{array}\right]$ & 33.69 & 0 & 0 \\
\hline I & $\left(\begin{array}{lll}1 & 1 & 2\end{array}\right)\left[\begin{array}{lll}1 & \overline{1} & 0\end{array}\right]$ & $\left(\begin{array}{lll}1 & 1 & 2\end{array}\right)\left[\begin{array}{lll}1 & \overline{1} & 0\end{array}\right]$ & 0 & 35.26 & 45 \\
\hline $\mathrm{J}$ & $\left(\begin{array}{lll}1 & 1 & 4\end{array}\right)\left[\begin{array}{lll}1 & \overline{1} & 0\end{array}\right]$ & $\left(\begin{array}{lll}1 & 1 & 4\end{array}\right)\left[\begin{array}{lll}1 & \overline{1} & 0\end{array}\right]$ & 0 & 19.47 & 45 \\
\hline $\mathrm{T}^{* \prime}$ & $\left(\begin{array}{lll}11 & 8 & 11\end{array}\right)\left[\begin{array}{lll}\overline{4} & 11 & \overline{4}\end{array}\right]$ & $\left(\begin{array}{lll}11 & 8 & 11\end{array}\right)\left[\begin{array}{lll}4 & \overline{11} & 4\end{array}\right]$ & 24.57 & 51.04 & 53.97 \\
\hline
\end{tabular}


Table 1 (contd.)

\begin{tabular}{|c|c|c|c|c|c|}
\hline \multirow[t]{2}{*}{ Code } & \multirow[t]{2}{*}{ (A) } & \multirow[t]{2}{*}{ (B) } & \multicolumn{3}{|c|}{ Euler angles } \\
\hline & & & $\phi_{1}$ & $\Phi$ & $\phi_{2}$ \\
\hline $\mathrm{E}^{\prime}$ & $\left(\begin{array}{lll}1 & 1 & 1\end{array}\right)\left[\begin{array}{lll}1 & 1 & 0\end{array}\right]$ & $\left(\begin{array}{lll}1 & 1 & 1\end{array}\right)\left[\begin{array}{lll}0 & \overline{1} & 1\end{array}\right]$ & 60 & 54.74 & 45 \\
\hline $\mathrm{E}$ & $\left(\begin{array}{lll}1 & 1 & 1\end{array}\right)\left[\begin{array}{lll}1 & \overline{1} & 0\end{array}\right]$ & $\left(\begin{array}{llll}1 & 1 & 1\end{array}\right)\left[\begin{array}{lll}1 & 1 & 0\end{array}\right]$ & 0 & 54.74 & 45 \\
\hline $\mathbf{F}$ & $\left(\begin{array}{lll}1 & 1 & 1\end{array}\right)\left[\begin{array}{lll}2 & 1 & 1\end{array}\right]$ & $\left(\begin{array}{lll}1 & 1 & 1\end{array}\right)\left[\begin{array}{lll}1 & \overline{2} & 1\end{array}\right]$ & 30 & 54.74 & 45 \\
\hline $\mathbf{F}^{\prime}$ & $\left(\begin{array}{llll}1 & 1 & 1\end{array}\right)\left[\begin{array}{lll}2 & 1 & 1\end{array}\right]$ & $\left(\begin{array}{lll}1 & 1 & 1\end{array}\right)\left[\begin{array}{lll}1 & 1 & 2\end{array}\right]$ & 90 & 54.74 & 45 \\
\hline $\mathbf{L}$ & $\left(\begin{array}{lll}0 & 1 & 1\end{array}\right)\left[\begin{array}{lll}0 & \overline{1} & 1\end{array}\right]$ & $\begin{array}{l}\left(\begin{array}{lll}0 & 1 & 1\end{array}\right)\left[\begin{array}{lll}0 & \overline{1} & 1\end{array}\right] \\
\left(\begin{array}{lll}1 & 0 & 1\end{array}\right)\left[\begin{array}{lll}\overline{1} & 0 & 1\end{array}\right]\end{array}$ & $\begin{array}{l}90 \\
90\end{array}$ & $\begin{array}{l}45 \\
45\end{array}$ & $\begin{array}{r}0 \\
90\end{array}$ \\
\hline
\end{tabular}

(b)

\begin{tabular}{|c|c|c|c|c|c|}
\hline \multirow[t]{2}{*}{ Code } & \multirow[t]{2}{*}{ (A) } & \multirow[t]{2}{*}{ (B) } & \multicolumn{3}{|c|}{ Euler Angles } \\
\hline & & & $\overline{\phi_{1}}$ & $\Phi$ & $\phi_{2}$ \\
\hline $\mathrm{C}$ & $\left(\begin{array}{lll}0 & 0 & 1\end{array}\right)\left[\begin{array}{lll}1 & 0 & 0\end{array}\right]$ & 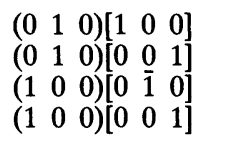 & $\begin{array}{r}0 \\
90 \\
0 \\
90\end{array}$ & $\begin{array}{l}90 \\
90 \\
90 \\
90\end{array}$ & $\begin{array}{r}0 \\
0 \\
90 \\
90\end{array}$ \\
\hline $\mathrm{CG}^{\prime}$ & $\left(\begin{array}{lll}0 & 2 & 1\end{array}\right)\left[\begin{array}{lll}1 & 0 & 0\end{array}\right]$ & 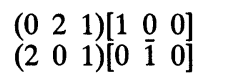 & $\begin{array}{l}0 \\
0\end{array}$ & $\begin{array}{l}63.43 \\
63.43\end{array}$ & $\begin{array}{r}0 \\
90\end{array}$ \\
\hline G & $\left(\begin{array}{lll}0 & 1 & 1\end{array}\right)\left[\begin{array}{lll}1 & 0 & 0\end{array}\right]$ & $\begin{array}{l}\left(\begin{array}{lll}0 & 1 & 1\end{array}\right)\left[\begin{array}{lll}1 & 0 & 0\end{array}\right] \\
\left(\begin{array}{llll}1 & 0 & 1\end{array}\right)\left[\begin{array}{lll}0 & 1 & 0\end{array}\right]\end{array}$ & $\begin{array}{l}0 \\
0 \\
\end{array}$ & $\begin{array}{l}45 \\
45 \\
\end{array}$ & $\begin{array}{r}0 \\
90 \\
\end{array}$ \\
\hline B & $\left(\begin{array}{lll}0 & 1 & 1\end{array}\right)\left[\begin{array}{lll}2 & \overline{1} & 1\end{array}\right]$ & 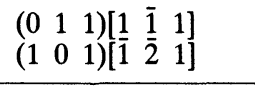 & $\begin{array}{l}35.26 \\
35.26 \\
\end{array}$ & $\begin{array}{l}45 \\
45 \\
\end{array}$ & $\begin{array}{r}0 \\
90\end{array}$ \\
\hline $\mathrm{S}_{3}^{\prime}$ & $\left(\begin{array}{lll}\overline{1} & \overline{2} & \overline{3}\end{array}\right)\left[\begin{array}{lll}\overline{6} & \overline{3} & 4\end{array}\right]$ & $\left(\begin{array}{lll}1 & 3 & 2\end{array}\right)\left[\begin{array}{lll}6 & \overline{4} & 3\end{array}\right]$ & 27.03 & 57.69 & 18.43 \\
\hline $\mathrm{T}^{\prime}$ & $\left(\begin{array}{lll}4 & 4 & 11\end{array}\right)\left[\begin{array}{lll}11 & 11 & 8\end{array}\right]$ & $\left(\begin{array}{lll}4 & 11 & 4\end{array}\right)\left[\begin{array}{lll}11 & \overline{8} & 11\end{array}\right]$ & 41.65 & 71.13 & 19.98 \\
\hline $\mathrm{Cu}^{\prime}$ & $\left(\begin{array}{lll}1 & 1 & 2\end{array}\right)\left[\begin{array}{lll}1 & 1 & 1\end{array}\right]$ & $\left(\begin{array}{llll}1 & 2 & 1\end{array}\right)\left[\begin{array}{lll}1 & \overline{1} & 1\end{array}\right]$ & 39.23 & 65.9 & 26.56 \\
\hline $\mathbf{H}$ & $\left(\begin{array}{lll}0 & 0 & 1\end{array}\right)\left[\begin{array}{lll}1 & 1 & 0\end{array}\right]$ & $\left.\begin{array}{lll}(0 & 1 & 0\end{array}\right)\left[\begin{array}{lll}1 & 0 & 1\end{array}\right]$ & $\begin{array}{l}45 \\
45 \\
\end{array}$ & $\begin{array}{l}90 \\
90 \\
\end{array}$ & $\begin{array}{r}0 \\
90 \\
\end{array}$ \\
\hline $\mathbf{K}^{\prime}$ & $\left(\begin{array}{lll}0 & 0 & 1\end{array}\right)\left[\begin{array}{lll}3 & 2 & 0\end{array}\right]$ & $\left.\begin{array}{lll}\left(\begin{array}{lll}0 & 1 & 0\end{array}\right)\left[\begin{array}{lll}2 & 0 & 3\end{array}\right] \\
(1 & 0 & 0\end{array}\right)\left[\begin{array}{lll}0 & 2 & 3\end{array}\right]$ & $\begin{array}{l}56.31 \\
56.31 \\
\end{array}$ & $\begin{array}{l}90 \\
90 \\
-\end{array}$ & $\begin{array}{r}0 \\
90 \\
-\end{array}$ \\
\hline $\mathbf{K}$ & $\left(\begin{array}{lll}0 & 0 & 1\end{array}\right)\left[\begin{array}{lll}3 & 2 & 0\end{array}\right]$ & 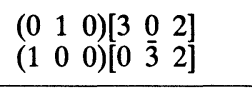 & $\begin{array}{l}33.69 \\
33.69 \\
\end{array}$ & $\begin{array}{l}90 \\
90 \\
\end{array}$ & $\begin{array}{r}0 \\
90 \\
\end{array}$ \\
\hline$I^{\prime}$ & $\left(\begin{array}{lll}1 & 1 & 2\end{array}\right)\left[\begin{array}{lll}\overline{1} & 1 & 0\end{array}\right]$ & $\left(\begin{array}{lll}2 & 1 & 1\end{array}\right)\left[\begin{array}{lll}0 & \overline{1} & 1\end{array}\right]$ & 50.77 & 65.9 & 63.43 \\
\hline
\end{tabular}


Table 1 (contd.)

\begin{tabular}{|c|c|c|c|c|c|}
\hline \multirow[t]{2}{*}{ Code } & \multirow[t]{2}{*}{ (A) } & \multirow[t]{2}{*}{ (B) } & \multicolumn{3}{|c|}{ Euler angles } \\
\hline & & & $\phi_{1}$ & $\Phi$ & $\phi_{2}$ \\
\hline $\mathrm{J}^{\prime}$ & $\left(\begin{array}{lll}1 & 1 & 4\end{array}\right)\left[\begin{array}{lll}\overline{1} & 1 & 0\end{array}\right]$ & $\left(\begin{array}{lll}4 & 1 & 1\end{array}\right)\left[\begin{array}{lll}0 & \overline{1} & 1\end{array}\right]$ & 46.69 & 76.37 & 75.96 \\
\hline $\mathrm{T}^{*}$ & 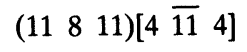 & $\left(\begin{array}{lll}11 & 8 & 11\end{array}\right)\left[\begin{array}{lll}4 & \overline{11} & 4\end{array}\right]$ & 24.57 & 51.04 & 53.97 \\
\hline$E^{\prime}$ & $\left(\begin{array}{lll}1 & 1 & 1\end{array}\right)\left[\begin{array}{lll}1 & 1 & 0\end{array}\right]$ & $\left(\begin{array}{lll}1 & 1 & 1\end{array}\right)\left[\begin{array}{lll}0 & \overline{1} & 1\end{array}\right]$ & 60 & 54.74 & 45 \\
\hline $\mathrm{E}$ & $\left(\begin{array}{llll}1 & 1 & 1\end{array}\right)\left[\begin{array}{lll}1 & 1 & 0\end{array}\right]$ & $\left(\begin{array}{lll}1 & 1 & 1\end{array}\right)\left[\begin{array}{lll}1 & 1 & 0\end{array}\right]$ & 0 & 54.74 & 45 \\
\hline $\mathbf{F}$ & $\left(\begin{array}{lll}1 & 1 & 1\end{array}\right)\left[\begin{array}{lll}2 & 1 & 1\end{array}\right]$ & $\left(\begin{array}{lll}1 & 1 & 1\end{array}\right)\left[\begin{array}{lll}1 & \overline{2} & 1\end{array}\right]$ & 30 & 54.74 & 45 \\
\hline $\mathbf{F}^{\prime}$ & $\left(\begin{array}{llll}1 & 1 & 1\end{array}\right)\left[\begin{array}{lll}2 & 1 & 1\end{array}\right]$ & $\left(\begin{array}{lllll}1 & 1 & 1\end{array}\right)\left[\begin{array}{lll}\overline{1} & \overline{1} & 2\end{array}\right]$ & 90 & 54.74 & 45 \\
\hline $\mathrm{L}$ & $\left(\begin{array}{lll}0 & 1 & 1\end{array}\right)\left[\begin{array}{lll}0 & 1 & 1\end{array}\right]$ & $\begin{array}{l}\left(\begin{array}{lll}0 & 1 & 1\end{array}\right)\left[\begin{array}{lll}0 & \overline{1} & 1\end{array}\right] \\
\left(\begin{array}{lll}1 & 0 & 1\end{array}\right)\left[\begin{array}{lll}\overline{1} & 0 & 1\end{array}\right]\end{array}$ & $\begin{array}{l}90 \\
90\end{array}$ & $\begin{array}{l}45 \\
45\end{array}$ & $\begin{array}{r}0 \\
90\end{array}$ \\
\hline
\end{tabular}

(c)

\begin{tabular}{|c|c|c|c|c|c|}
\hline \multirow[t]{2}{*}{ Code } & \multirow[t]{2}{*}{ (A) } & \multirow[t]{2}{*}{ (B) } & \multicolumn{3}{|c|}{ Euler angles } \\
\hline & & & $\phi_{1}$ & $\Phi$ & $\phi_{2}$ \\
\hline $\mathrm{C}$ & $\left(\begin{array}{lll}0 & 0 & 1\end{array}\right)\left[\begin{array}{lll}1 & 0 & 0\end{array}\right]$ & 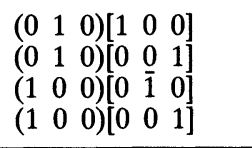 & $\begin{array}{r}0 \\
90 \\
0 \\
90\end{array}$ & $\begin{array}{l}90 \\
90 \\
90 \\
90\end{array}$ & $\begin{array}{r}0 \\
0 \\
90 \\
90\end{array}$ \\
\hline $\mathrm{CG}^{\prime}$ & $\left(\begin{array}{lll}0 & 2 & 1\end{array}\right)\left[\begin{array}{lll}1 & 0 & 0\end{array}\right]$ & $\left(\begin{array}{lll}2 & 1 & 0\end{array}\right)\left[\begin{array}{lll}0 & 0 & 1\end{array}\right]$ & 90 & 90 & 63.43 \\
\hline $\mathrm{CG}$ & $\left(\begin{array}{lll}0 & 2 & 1\end{array}\right)\left[\begin{array}{lll}1 & 0 & 0\end{array}\right]$ & $\left(\begin{array}{lll}1 & 2 & 0\end{array}\right)\left[\begin{array}{lll}0 & 0 & 1\end{array}\right]$ & 90 & 90 & 26.56 \\
\hline G & $\left(\begin{array}{lll}0 & 1 & 1\end{array}\right)\left[\begin{array}{lll}1 & 0 & 0\end{array}\right]$ & $\left(\begin{array}{lll}1 & 1 & 0\end{array}\right)\left[\begin{array}{lll}0 & 0 & 1\end{array}\right]$ & 90 & 90 & 45 \\
\hline $\mathbf{B}^{\prime}$ & $\left(\begin{array}{lll}0 & \overline{1} & \overline{1}\end{array}\right)\left[\begin{array}{lll}2 & \overline{1} & 1\end{array}\right]$ & $\left(\begin{array}{lll}1 & 1 & 0\end{array}\right)\left[\begin{array}{lll}1 & 1 & 2\end{array}\right]$ & 54.74 & 90 & 45 \\
\hline $\mathrm{S}_{3}^{\prime \prime}$ & $\left(\begin{array}{lll}1 & 2 & 3\end{array}\right)\left[\begin{array}{lll}6 & 3 & 4\end{array}\right]$ & $\left(\begin{array}{lll}2 & 3 & 1\end{array}\right)\left[\begin{array}{lll}3 & \overline{4} & 6\end{array}\right]$ & 52.87 & 74.5 & 33.69 \\
\hline $\mathrm{T}^{\prime}$ & $\left(\begin{array}{lll}4 & 4 & 11\end{array}\right)\left[\begin{array}{lll}11 & 11 & 8\end{array}\right]$ & $\left(\begin{array}{lll}4 & 11 & 4\end{array}\right)\left[\begin{array}{lll}11 & \overline{8} & 11\end{array}\right]$ & 41.65 & 71.13 & 19.98 \\
\hline $\mathrm{Cu}^{\prime}$ & $\left(\begin{array}{lll}1 & 1 & 2\end{array}\right)\left[\begin{array}{lll}1 & 1 & 1\end{array}\right]$ & $\left(\begin{array}{lll}1 & 2 & 1\end{array}\right)\left[\begin{array}{lll}1 & 1 & 1\end{array}\right]$ & 39.23 & 65.9 & 26.56 \\
\hline $\mathbf{H}$ & $\left(\begin{array}{lll}0 & 0 & 1\end{array}\right)\left[\begin{array}{lll}1 & 1 & 0\end{array}\right]$ & $\left.\begin{array}{lll}(0 & 1 & 0\end{array}\right)\left[\begin{array}{lll}1 & 0 & 1\end{array}\right]$ & $\begin{array}{l}45 \\
45\end{array}$ & $\begin{array}{l}90 \\
90\end{array}$ & $\begin{array}{r}0 \\
90\end{array}$ \\
\hline $\mathbf{K}^{\prime}$ & $\left(\begin{array}{lll}0 & 0 & 1\end{array}\right)\left[\begin{array}{lll}3 & 2 & 0\end{array}\right]$ & $\left(\begin{array}{lll}0 & 1 & 0\end{array}\right)\left[\begin{array}{lll}2 & 0 & 3\end{array}\right]$ & $\begin{array}{l}56.31 \\
56.31\end{array}$ & $\begin{array}{l}90 \\
90\end{array}$ & $\begin{array}{r}0 \\
90\end{array}$ \\
\hline $\mathrm{K}$ & $\left(\begin{array}{lllll}0 & 0 & 1\end{array}\right)\left(\begin{array}{lll}3 & 2 & 0\end{array}\right]$ & $\left.\begin{array}{lll}\left(\begin{array}{llll}0 & 1 & 0\end{array}\right)\left[\begin{array}{lll}3 & 0 & 2\end{array}\right] \\
(1 & 0 & 0\end{array}\right)\left[\begin{array}{lll}0 & \overline{3} & 2\end{array}\right]$ & $\begin{array}{l}33.69 \\
33.69\end{array}$ & $\begin{array}{l}90 \\
90\end{array}$ & $\begin{array}{r}0 \\
90\end{array}$ \\
\hline
\end{tabular}


Table 1 (contd.)

\begin{tabular}{|c|c|c|c|c|c|}
\hline \multirow[t]{2}{*}{ Code } & \multirow[t]{2}{*}{ (A) } & \multirow[t]{2}{*}{ (B) } & \multicolumn{3}{|c|}{ Euler angles } \\
\hline & & & $\phi_{1}$ & $\Phi$ & $\phi_{2}$ \\
\hline $\mathrm{I}^{\prime}$ & $\left(\begin{array}{lll}1 & 1 & 2\end{array}\right)\left[\begin{array}{lll}\overline{1} & 1 & 0\end{array}\right]$ & $\left(\begin{array}{lll}2 & 1 & 1\end{array}\right)\left[\begin{array}{lll}0 & \overline{1} & 1\end{array}\right]$ & 50.77 & 65.9 & 63.43 \\
\hline $\mathrm{J}^{\prime}$ & $\left(\begin{array}{lll}1 & 1 & 4\end{array}\right)\left[\begin{array}{lll}1 & 1 & 0\end{array}\right]$ & $\left(\begin{array}{lll}4 & 1 & 1\end{array}\right)\left[\begin{array}{lll}0 & 1 & 1\end{array}\right]$ & 46.69 & 76.37 & 75.96 \\
\hline $\mathrm{T}^{*}$ & $\left(\begin{array}{lll}11 & 8 & 11\end{array}\right)\left[\begin{array}{lll}4 & \overline{11} & 4\end{array}\right]$ & $\left(\begin{array}{lll}11 & 11 & 8\end{array}\right)\left[\begin{array}{lll}\overline{4} & \overline{4} & 11\end{array}\right]$ & 90 & 62.78 & 45 \\
\hline$E^{\prime}$ & $\left(\begin{array}{lll}1 & 1 & 1\end{array}\right)\left[\begin{array}{lll}\overline{1} & 1 & 0\end{array}\right]$ & $\left(\begin{array}{lll}1 & 1 & 1\end{array}\right)\left[\begin{array}{lll}0 & \overline{1} & 1\end{array}\right]$ & 60 & 54.74 & 45 \\
\hline $\mathrm{E}$ & $\left(\begin{array}{lll}1 & 1 & 1\end{array}\right)\left[\begin{array}{lll}1 & 1 & 0\end{array}\right]$ & $\left(\begin{array}{lll}1 & 1 & 1\end{array}\right)\left[\begin{array}{lll}1 & 1 & 0\end{array}\right]$ & 0 & 54.74 & 45 \\
\hline $\mathrm{F}$ & $\left(\begin{array}{lll}1 & 1 & 1\end{array}\right)\left[\begin{array}{lll}\overline{2} & 1 & 1\end{array}\right]$ & $\left(\begin{array}{lll}1 & 1 & 1\end{array}\right)\left[\begin{array}{lll}1 & \overline{2} & 1\end{array}\right]$ & 30 & 54.74 & 45 \\
\hline $\mathrm{F}^{\prime}$ & $\left(\begin{array}{lll}1 & 1 & 1\end{array}\right)\left[\begin{array}{lll}2 & \overline{1} & \overline{1}\end{array}\right]$ & $\left(\begin{array}{lll}1 & 1 & 1\end{array}\right)\left[\begin{array}{lll}1 & 1 & 2\end{array}\right]$ & 90 & 54.74 & 45 \\
\hline $\mathbf{L}$ & $\left(\begin{array}{lll}0 & 1 & 1\end{array}\right)\left[\begin{array}{lll}0 & \overline{1} & 1\end{array}\right]$ & $\left(\begin{array}{lll}1 & 1 & 0\end{array}\right)\left[\begin{array}{lll}1 & \overline{1} & 0\end{array}\right]$ & 0 & 90 & 45 \\
\hline
\end{tabular}

( $x_{3}$ is the twofold axis). Only the range for $\phi_{1}$ would change, now becoming $0^{\circ}-180^{\circ}$. This case would apply to shear textures, with $\mathrm{x}_{1}$ as shear direction and $x_{2}$ as shear plane normal. Table 2 lists the Euler angles for unit zone 3 of some important crystal orientations.

\section{DISCUSSION}

Wenk and Kocks (1986) have proposed to replace conventional Euler diagrams of O.D.F. by figures in which $\phi_{2}$ and $\Phi$ act as polar coordinates instead of Cartesian coordinates (sections of $\phi_{1}=$ constant). A figure such as Figure 6 would then look like a set of 19 inverse pole figures (one for each value of $\phi_{1}$, with steps of $5^{\circ}$ for $\phi_{1}$ ), each taking the aspect of Figure 4. It is true that these authors prefer to use the equal area projection method instead of the stereographic projection method (see below). A "true" inverse pole figure showing the density distribution of the $\mathrm{x}_{3}$ axes is then obtained by averaging the density distributions of the $19 \phi_{1}$-sections representing the O.D.F.

Conversely, these authors also propose to use $\phi_{1}$ and $\Phi$ as polar coordinates in $\phi_{2}=$ constant sections. An Euler diagram such as Figure 2 would then be replaced by a set of 19 equal area projections of a portion of a sphere. Making the average of these 19 density distribution would then lead to an equal area $\{100\}$ pole 
Table 2 Euler angles of a number of texture components of torsion (or simple shear) textures of cubic metals. $x_{1}$ is tangential to the sample (shear direction), $x_{2}$ is parallel to the sample axis (shear plane normal) and $x_{3}$ is the radial direction. The latter is a twofold symmetry axis of the sample for such a texture. Representations for which $x_{3}$ lies in unit zone 3, consisting of the triangles $(010)-(110)-(111)$ and (100)-(110)-(111).

Convention for the indicial notations: (shear plane normal) [shear direction].

Column (A) lists crystal orientations that are all physically different from each other. They are separated by a full line.

The indicial notations of column (B) represent the same crystal orientations as those of column (A). Among the 24 possible descriptions of a given orientation, those for which the $x_{3}$-axis falls within the considered unit zone are given. Two indicial notations of column (B) that are not separated by a full line represent the same crystal orientation

\begin{tabular}{|c|c|c|c|c|c|}
\hline \multirow[t]{2}{*}{ Code } & \multirow[t]{2}{*}{ (A) } & \multirow[t]{2}{*}{ (B) } & \multicolumn{3}{|c|}{ Euler angles } \\
\hline & & & $\phi_{1}$ & $\Phi$ & $\phi_{2}$ \\
\hline $\mathrm{C}$ & $\left(\begin{array}{lll}0 & 0 & 1\end{array}\right)\left[\begin{array}{lll}1 & 0 & 0\end{array}\right]$ & 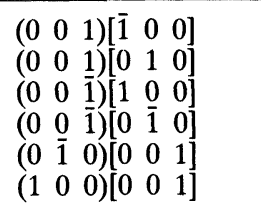 & $\begin{array}{r}180 \\
180 \\
0 \\
0 \\
90 \\
90\end{array}$ & $\begin{array}{l}90 \\
90 \\
90 \\
90 \\
90 \\
90\end{array}$ & $\begin{array}{r}0 \\
90 \\
0 \\
90 \\
90 \\
0\end{array}$ \\
\hline $\mathrm{CG}^{\prime}$ & $\left(\begin{array}{lll}0 & 2 & 1\end{array}\right)\left[\begin{array}{lll}1 & 0 & 0\end{array}\right]$ & $\left(\begin{array}{lll}1 & \overline{2} & 0\end{array}\right)\left[\begin{array}{lll}0 & 0 & 1\end{array}\right]$ & 90 & 90 & 63.43 \\
\hline CG & $\left(\begin{array}{lll}0 & 2 & 1\end{array}\right)\left[\begin{array}{lll}\overline{1} & 0 & 0\end{array}\right]$ & $\left(\begin{array}{lll}2 & \overline{1} & 0\end{array}\right)\left[\begin{array}{lll}0 & 0 & 1\end{array}\right]$ & 90 & 90 & 26.56 \\
\hline G & $\left(\begin{array}{lll}0 & 1 & 1\end{array}\right)\left[\begin{array}{lll}1 & 0 & 0\end{array}\right]$ & $\left(\begin{array}{lll}1 & \overline{1} & 0\end{array}\right)\left[\begin{array}{lll}0 & 0 & 1\end{array}\right]$ & 90 & 90 & 45 \\
\hline B & $\left(\begin{array}{lll}0 & 1 & 1\end{array}\right)\left[\begin{array}{lll}2 & \overline{1} & 1\end{array}\right]$ & $\left(\begin{array}{lll}0 & \overline{1} & 1\end{array}\right)\left[\begin{array}{lll}\overline{2} & 1 & 1\end{array}\right]$ & $\begin{array}{r}150 \\
30\end{array}$ & $\begin{array}{l}54.74 \\
54.74\end{array}$ & $\begin{array}{l}45 \\
45\end{array}$ \\
\hline $\mathbf{B}^{\prime}$ & $\left(\begin{array}{lll}0 & \overline{1} & \overline{1}\end{array}\right)\left[\begin{array}{lll}2 & \overline{1} & 1\end{array}\right]$ & $\left(\begin{array}{lll}1 & \overline{1} & 0\end{array}\right)\left[\begin{array}{lll}\overline{1} & \overline{1} & 2\end{array}\right]$ & 90 & 54.74 & 45 \\
\hline $\mathrm{S}_{3}$ & $\left(\begin{array}{lll}\overline{1} & \overline{2} & \overline{3}\end{array}\right)\left[\begin{array}{lll}6 & 3 & \overline{4}\end{array}\right]$ & $\left(\begin{array}{lll}1 & \overline{2} & 3\end{array}\right)\left[\begin{array}{lll}\overline{6} & 3 & 4\end{array}\right]$ & 147.4 & 72.06 & 37.69 \\
\hline $\mathrm{S}_{3}^{\prime}$ & $\left(\begin{array}{lll}\overline{1} & \overline{2} & \overline{3}\end{array}\right)\left[\begin{array}{lll}\overline{6} & \overline{3} & 4\end{array}\right]$ & $\left(\begin{array}{lll}2 & \overline{1} & \overline{3}\end{array}\right)\left[\begin{array}{lll}3 & \overline{6} & 4\end{array}\right]$ & 32.57 & 72.06 & 52.31 \\
\hline $\mathrm{T}^{\prime}$ & $\left(\begin{array}{lll}4 & 4 & 11\end{array}\right)\left[\begin{array}{lll}11 & 11 & 8\end{array}\right]$ & $\left(\begin{array}{llll}4 & \overline{4} & \overline{11}\end{array}\right)\left[\begin{array}{lll}11 & \overline{11} & 8\end{array}\right]$ & 27.21 & 90 & 45 \\
\hline $\mathrm{T}$ & $\left(\begin{array}{lll}4 & 4 & 11\end{array}\right)\left[\begin{array}{lll}\overline{11} & \overline{11} & 8\end{array}\right]$ & $\left(\begin{array}{llll}4 & \overline{4} & 11\end{array}\right)\left[\begin{array}{lll}\overline{11} & 11 & 8\end{array}\right]$ & 152.78 & 90 & 45 \\
\hline $\mathrm{Cu}^{\prime}$ & $\left(\begin{array}{lll}1 & 1 & 2\end{array}\right)\left[\begin{array}{lll}1 & 1 & 1\end{array}\right]$ & $\left(\begin{array}{lll}1 & \overline{1} & \overline{2}\end{array}\right)\left[\begin{array}{lll}1 & \overline{1} & 1\end{array}\right]$ & 35.26 & 90 & 45 \\
\hline $\mathrm{Cu}$ & $\left(\begin{array}{lll}1 & 1 & 2\end{array}\right)\left[\begin{array}{lll}1 & \overline{1} & 1\end{array}\right]$ & $\left(\begin{array}{lll}1 & 1 & 2\end{array}\right)\left[\begin{array}{lll}\overline{1} & 1 & 1\end{array}\right]$ & 144.74 & 90 & 45 \\
\hline $\mathrm{H}$ & $\left(\begin{array}{lll}0 & 0 & 1\end{array}\right)\left[\begin{array}{lll}1 & 1 & 0\end{array}\right]$ & $\left(\begin{array}{lll}0 & 0 & 1\end{array}\right)\left[\begin{array}{lll}1 & 1 & 0\end{array}\right]$ & $\begin{array}{r}180 \\
0 \\
\end{array}$ & $\begin{array}{l}90 \\
90 \\
\end{array}$ & $\begin{array}{l}45 \\
45 \\
\end{array}$ \\
\hline
\end{tabular}


Table 2 (contd.)

\begin{tabular}{|c|c|c|c|c|c|}
\hline \multirow[t]{2}{*}{ Code } & \multirow[t]{2}{*}{ (A) } & \multirow[t]{2}{*}{ (B) } & \multicolumn{3}{|c|}{ Euler angles } \\
\hline & & & $\phi_{1}$ & $\Phi$ & $\phi_{2}$ \\
\hline $\mathbf{K}^{\prime}$ & $\left(\begin{array}{lll}0 & 0 & 1\end{array}\right)\left[\begin{array}{lll}3 & 2 & 0\end{array}\right]$ & $\begin{array}{l}\left(\begin{array}{lll}0 & 0 & 1\end{array}\right)\left[\begin{array}{lll}2 & 3 & 0\end{array}\right] \\
\left(\begin{array}{lll}0 & 0 & 1\end{array}\right)\left[\begin{array}{lll}3 & 2 & 0\end{array}\right]\end{array}$ & $\begin{array}{r}180 \\
0\end{array}$ & $\begin{array}{l}90 \\
90\end{array}$ & $\begin{array}{l}56.31 \\
33.69\end{array}$ \\
\hline $\mathrm{K}$ & $\left(\begin{array}{lll}0 & 0 & \overline{1}\end{array}\right)\left[\begin{array}{lll}3 & 2 & 0\end{array}\right]$ & $\begin{array}{l}\left(\begin{array}{lll}0 & 0 & \overline{1}\end{array}\right)\left[\begin{array}{lll}2 & \overline{3} & 0\end{array}\right] \\
\left(\begin{array}{llll}0 & 0 & 1\end{array}\right)\left[\begin{array}{lll}\overline{3} & 2 & 0\end{array}\right]\end{array}$ & $\begin{array}{r}0 \\
180\end{array}$ & $\begin{array}{l}90 \\
90\end{array}$ & $\begin{array}{l}56.31 \\
33.69\end{array}$ \\
\hline$I^{\prime}$ & $\left(\begin{array}{lll}1 & 1 & 2\end{array}\right)\left[\begin{array}{lll}\overline{1} & 1 & 0\end{array}\right]$ & $\begin{array}{l}\left(\begin{array}{lll}1 & 1 & \overline{2}\end{array}\right)\left[\begin{array}{lll}1 & \overline{1} & 0\end{array}\right] \\
\left(\begin{array}{lll}1 & \overline{2} & 1\end{array}\right)\left[\begin{array}{lll}\overline{1} & 0 & 1\end{array}\right]\end{array}$ & $\begin{array}{r}0 \\
120\end{array}$ & $\begin{array}{l}54.74 \\
54.74\end{array}$ & $\begin{array}{l}45 \\
45\end{array}$ \\
\hline I & $\left(\begin{array}{lll}1 & 1 & 2\end{array}\right)\left[\begin{array}{lll}1 & \overline{1} & 0\end{array}\right]$ & 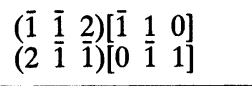 & $\begin{array}{r}180 \\
60\end{array}$ & $\begin{array}{l}54.74 \\
54.74\end{array}$ & $\begin{array}{l}45 \\
45\end{array}$ \\
\hline $\mathrm{J}^{\prime}$ & $\left(\begin{array}{lll}1 & 1 & 4\end{array}\right)\left[\begin{array}{lll}1 & 1 & 0\end{array}\right]$ & $\left(\begin{array}{lll}1 & 1 & \overline{4}\end{array}\right)\left[\begin{array}{lll}1 & \overline{1} & 0\end{array}\right]$ & 0 & 70.53 & 45 \\
\hline $\mathbf{J}$ & $\left(\begin{array}{lll}1 & 1 & 4\end{array}\right)\left[\begin{array}{lll}1 & \overline{1} & 0\end{array}\right]$ & $\left(\begin{array}{lll}\overline{1} & \overline{1} & 4\end{array}\right)\left[\begin{array}{lll}\overline{1} & 1 & 0\end{array}\right]$ & 180 & 70.53 & 45 \\
\hline $\mathrm{T}^{*}$ & $\left(\begin{array}{lll}11 & 8 & 11\end{array}\right)\left[\begin{array}{lll}4 & \overline{11} & 4\end{array}\right]$ & $\left(\begin{array}{lll}11 & \overline{11} & \overline{8}\end{array}\right)\left[\begin{array}{lll}4 & \overline{4} & 11\end{array}\right]$ & 62.78 & 90 & 45 \\
\hline $\mathrm{T}^{* \prime}$ & $\left(\begin{array}{lll}11 & 8 & 11\end{array}\right)\left[\begin{array}{lll}\overline{4} & 11 & \overline{4}\end{array}\right]$ & $\left(\begin{array}{lll}11 \overline{11} & 8\end{array}\right)\left[\begin{array}{lll}\overline{4} & 4 & 11\end{array}\right]$ & 117.21 & 90 & 45 \\
\hline $\mathrm{E}^{\prime}$ & $\left(\begin{array}{lll}1 & 1 & 1\end{array}\right)\left[\begin{array}{lll}1 & 1 & 0\end{array}\right]$ & $\left(\begin{array}{lll}1 & \overline{1} & 1\end{array}\right)\left[\begin{array}{lll}\overline{1} & 0 & 1\end{array}\right]$ & 129.2 & 65.90 & 26.56 \\
\hline $\mathbf{E}$ & $\left(\begin{array}{lll}1 & 1 & 1\end{array}\right)\left[\begin{array}{lll}1 & \overline{1} & 0\end{array}\right]$ & $\left(\begin{array}{lll}1 & \overline{1} & \overline{1}\end{array}\right)\left[\begin{array}{lll}0 & \overline{1} & 1\end{array}\right]$ & 50.77 & 65.90 & 63.43 \\
\hline $\mathbf{F}$ & $\left(\begin{array}{lll}1 & 1 & 1\end{array}\right)\left[\begin{array}{lll}2 & 1 & 1\end{array}\right]$ & $\left(\begin{array}{lll}1 & \overline{1} & \overline{1}\end{array}\right)\left[\begin{array}{lll}1 & \overline{1} & 2\end{array}\right]$ & 54.74 & 90 & 45 \\
\hline $\mathrm{F}^{\prime}$ & $\left(\begin{array}{lll}1 & 1 & 1\end{array}\right)\left[\begin{array}{lll}2 & \overline{1} & 1\end{array}\right]$ & $\left(\begin{array}{lll}1 & \overline{1} & 1\end{array}\right)\left[\begin{array}{lll}\overline{1} & 1 & 2\end{array}\right]$ & 125.26 & 90 & 45 \\
\hline $\mathbf{L}$ & $\left(\begin{array}{lll}0 & 1 & 1\end{array}\right)\left[\begin{array}{lll}0 & \overline{1} & 1\end{array}\right]$ & 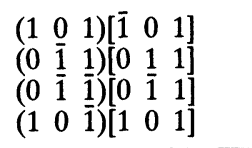 & $\begin{array}{r}135 \\
135 \\
45 \\
45\end{array}$ & $\begin{array}{l}90 \\
90 \\
90 \\
90\end{array}$ & $\begin{array}{r}0 \\
90 \\
90 \\
0\end{array}$ \\
\hline
\end{tabular}

figure, which can be easily understood since the orientation of the [001] axis with respect to the sample axes only depends on $\phi_{1}$ and $\Phi$ (see Figure 1).

This method has the following advantages:

- the representation of the $(001)$-pole $(\Phi=0)$ no longer degenerates into a line segment;

-it becomes possible to estimate the importance of a texture component quantitatively by a visual inspection of the graphical 
representation of the O.D.F., since equal areas in the figure correspond to equal areas in orientation space;

- a comparably easy method is suggested to "see" the correspondence between the O.D.F. and the $\{100\}$ or inverse pole figures of the texture.

One of the disadvantages of the conventional Euler diagrams is however retained: more than one "minimum range" is shown in the new diagrams (6 in the original paper), which may also lead to confusion when trying to estimate the importance of texture components. In addition, the new method has a few other disadvantages:

- a singularity of another type is introduced at the centres of the polar plots. Indeed, at $\Phi=0^{\circ}$, the value of the texture function $f(g)$ only depends of the sum $\phi_{1}+\phi_{2}$, not on the individual values of the $\phi_{1}$ and $\phi_{2}$ angles. Suppose for example a $\phi_{1}=10^{\circ}$ section. In a conventional Euler diagram, $\phi_{2}$ would range from $0^{\circ}$ to $90^{\circ}$ and therefore, along the line $\Phi=0^{\circ}, f(g)$ would take all the different values that correspond to the range $10^{\circ}-100^{\circ}$ of the sum $\phi_{1}+\phi_{2}$. In the type of plot that has been proposed by Wenk and Kocks, this line is represented by a single point (the centre), in which $f(g)$ should hence be multivalued. The authors have proposed to attribute the value $0^{\circ}$ conventionally to the angle $\phi_{2}$ at that point (the angle $\phi_{2}$ is also multivalued there). Suppose that by such convention, one attributes the value that $f(g)$ has at $\Phi=\phi_{2}=0^{\circ}$ and $\phi_{1}=10^{\circ}$ to that point. In that case, no discontinuity would be felt upon reaching $\Phi=0^{\circ}$ when traveling along a line $\phi_{2}=0^{\circ}$ in such a plot; but a discontinuity would still be felt when the point is approached along a line $\phi_{2}=\mathrm{C}$ for which $\mathrm{C}$ is different from zero. Since it is anyway impossible to represent all the multiple values of $f(g)$ at this singular point, it is no longer possible to distinguish between crystal orientations for which the sum $\phi_{1}+\phi_{2}$ takes different values at $\Phi=0^{\circ}$, such as (001)[100] and (001)[110]. This is a serious drawback, especially for noncubic crystal symmetries. In the case of cubic symmetry, the orientations for which $\Phi=0$ have crystallographic equivalent 
representations at $\Phi=90^{\circ}$ (in unit zone 3), where no ambiguities arise;

- measuring the angles $\phi_{1}, \Phi$ and $\phi_{2}$ is no longer easy;

- the equal area projections are unconventional for metallurgists, who are used to the stereographic projection for the representation of pole figures and inverse pole figures. An advantage of the stereographic projection is, that it respects the angles between intersecting circles on the orientation sphere;

- yet another type of O.D.F. representation is introduced, in addition to those that already exist (see e.g. Bunge (1982)). There is a strong need for standardization instead.

Before proposing a method in order to overcome most of these difficulties, it is useful to look at the area distortions that conventional projection methods introduce. Let such distortions be described by the ratio between the area as seen in one of the graphical representations, and the area in "true" orientation space. This ratio should not vary very much in the range used for inspection of the texture function.

In a equal area projection, the ratio is always equal to 1 . In a conventional Euler diagram, it is given by:

$$
R_{E}=\frac{1}{\sin \Phi}
$$

In a stereographic projection, it would be:

$$
R_{S}=\frac{1}{(1+\cos \Phi)^{2}}
$$

Table 3 shows some values of $R_{E}$ and $R_{S}$ as a function of $\Phi$ :

Table 3 Area ratios for Euler diagrams and stereographic projections

\begin{tabular}{ccc}
\hline$\Phi$ & $R_{E}$ & $R_{S}$ \\
\hline $0^{\circ}$ & $\infty$ & 0.25 \\
$45^{\circ}$ & 1.414 & 0.343 \\
$54.74^{\circ}$ & 1.225 & 0.402 \\
$90^{\circ}$ & 1 & 1 \\
\hline
\end{tabular}


Wenk and Kocks are quite right when they consider the variation of both $R_{E}$ and $R_{S}$ between $\Phi=0$ and $\Phi=90^{\circ}$ as untolerable. But what if we would deliberately restrict our inspection of the graphical representation of $f(g)$ to one minimum range only (also to avoid confusion when counting the number of texture components)? Let us first consider unit zone 1 of Table 1 . The range of $\Phi$ then is from $0^{\circ}$ to $54.74^{\circ}$. Table 3 shows that the variation of $R_{E}$ still is unacceptable. The maximal variation of $R_{S}$ is $61 \%$, which also is quite high and may lead to erroneous estimations of the importance of a texture component.

Mecking (1986) proposed to use unit zone 3 (see Table 1c or the shaded area in Figure 5) instead. The range of $\Phi$ now is from $54.74^{\circ}$ to $90^{\circ}$. The maximal variation of $R_{S}$ is $148 \%$, which is much to high. However, the variation of $R_{E}$ only is $22.5 \%$, which seems quite acceptable to the present author when it comes to visual evaluation of texture components. This leads to the following proposal:

- to go on representing texture functions by means of conventional Euler diagrams;

- taking care to make the boundaries between the unit zones 1,2 and 3 (as indicated in Table 1) visible, as for example has been done by means of the dashed lines in Figures 2 and 6;

- only look at unit zone 3 when analyzing a texture function. The range for $\mathrm{x}_{3}$ associated to this unit zone consists of the adjacent triangles $010-110-111$ and $100-110-111$; the shaded area of Figure 5 shows this unit zone in an Euler diagram. The range for $\phi_{1}$ is from $0^{\circ}$ to $90^{\circ}$ as usual.

This proposal at least avoids the introduction of a completely new type of O.D.F. representation, which would be just another obstacle to standardization. Other advantages are:

- within unit zone 3 , the are distortion never exceeds $22.5 \%$ (compared to $\infty$ in the conventional range consisting of the three unit zones together);

- there is no singular point;

- in unit zone 3, the relevant information is only contained once;

- it is possible to have an idea of the aspect of the axial densities within the 100-010-111 range of an inverse pole figure for $x_{3}$, by 


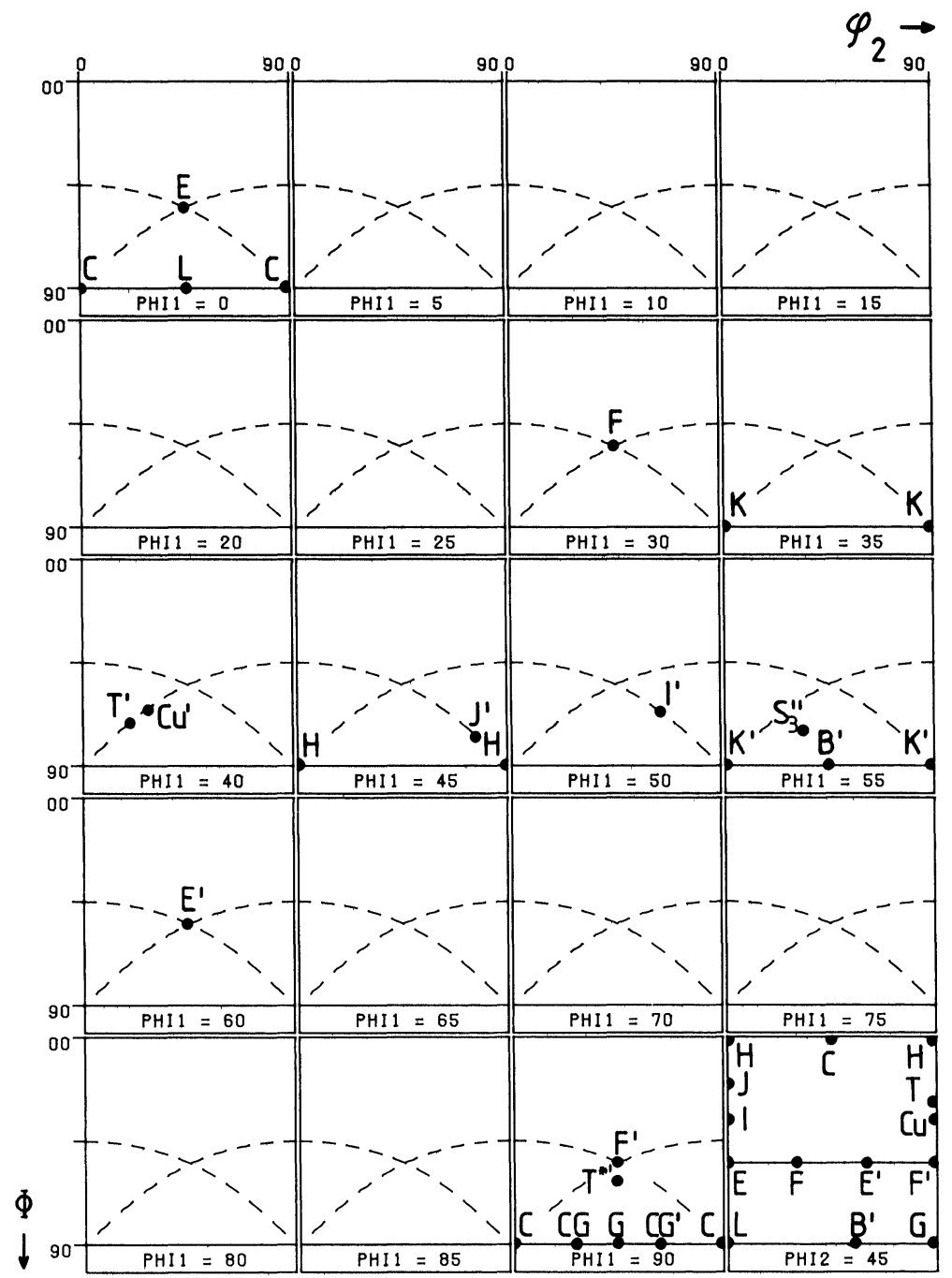

Figure 6 Euler diagram consisting of $19 \phi_{1}=$ constant sections and a $\phi_{2}=45^{\circ}$ section. The zone 3 representation of various frequently used crystal orientations are shown (case of cubic crystal symmetry), except in the $\phi_{2}=45^{\circ}$ section, where zone 1 and zone 2 representations are also shown. 
averaging the density distributions within unit zone 3 for the various $\phi_{1}$-sections in the range $0 \leq \phi_{1} \leq 90^{\circ}$; comparison of Figures 4 and 5 gives the point-to-point correspondence between the Euler diagram and the inverse pole figure.

- it remains easy to measure the Euler angles of a particular spot in the diagram.

Of the zone 3 orientations listed in Table 1c, those that are of interest for f.c.c. metals are shown in Figure 2. They are all shown in Figure 6, which contains 19 sections $\phi_{1}=$ constant with in addition the $\phi_{2}=45^{\circ}$ section, in which many of the orientations listed in Table $1 a-b-c$ can be seen.

\section{CONCLUSION}

Most objections against the use of the conventional Euler diagrams, as defined by Eqs. $(2 \mathrm{a}-\mathrm{c})$, do not apply any more when one restricts ones attention to unit zone 3 . This is a range for $\phi_{2}$ and $\Phi$ which in the case of cubic crystals is given by the shaded area of Figure 4; the range for $\phi_{1}$ remains from $0^{\circ}$ to $90^{\circ}$ (orthorombic sample symmetry, which is the usual case for rolling or annealing textures). It is therefore advised to show the limits of unit zone 3 in all Euler diagrams in order to facilitate interpretation.

It is possible to define the range of unit zone 3 also for other sample symmetries.

\section{References}

Bunge, H. J. (1982). Texture Analysis in Material Science, Butterworths, London.

Gil Sevillano, J., Van Houtte, P. and Aernoudt, E. (1980). Progr. Mater. Sci. 25, 69-412.

Hansen, J., Pospiech, J. and Lücke, K. (1978). Tables for Texture Analysis of Cubic Crystals, Springer, Berlin.

Mecking, H. (1986). Private Communication.

Pospiech, J. (1972). Kristall und Technik 7, 1057.

Van Houtte, P. and Aernoudt, E. (1976). Mater. Sci. Eng. 23, 11.

Wenk, H. R. and Kocks, U. F. (1986). The Representation of Orientation Distributions, Met. Trans., in press. 